\title{
DENSIDADE, POROSIDADE E RESISTÊNCIA À PENETRAÇÃO EM LATOSSOLO CULTIVADO SOB DIFERENTES SISTEMAS DE PREPARO DO SOLO
}

\author{
Cássio Antonio Tormena ${ }^{1,3 *}$; Mauro Cezar Barbosa ${ }^{2}$; Antônio Carlos Saraiva da Costa ${ }^{1}$; Antonio \\ Carlos Andrade Gonçalves ${ }^{1}$ \\ ${ }_{2}^{1}$ Depto de Agronomia - UEM, Av. Colombo, 5790 - CEP: 87020-900 - Maringá, PR. \\ Bolsista IC/CNPq. \\ ${ }^{3}$ Bolsista CNPq \\ *Autor correspondente <catormena@uem.br>
}

\begin{abstract}
RESUMO: Os sistemas de preparo e manejo do solo determinam as condições físicas para o crescimento das plantas e produtividade das culturas. O objetivo deste estudo é o de avaliar algumas propriedades físicas de um Latossolo Vermelho distrófico, cultivado com mandioca, após dois anos de implantação em diferentes sistemas de preparo do solo. Os tratamentos utilizados foram: Plantio Direto (mobilização do solo somente ao longo das linhas de plantio); Preparo Mínimo (escarificação a 0,30 m, seguido de gradagem niveladora) e Preparo Convencional (aração, com arado de aiveca a uma profundidade de 0,25 m, seguido de gradagem niveladora). Avaliaram-se, nas camadas de 0-0,10 m e 0,10-0,20 m, as seguintes propriedades físicas do solo: densidade, volume de macroporos, microporos, a porosidade e a resistência do solo à penetração das raízes, a cada 0,05 $\mathrm{m}$, até à profundidade de $0,40 \mathrm{~m}$, em três diferentes épocas. Constataramse maiores valores de densidade do solo e menores valores de macroporosidade na camada de 0-0,10 m no plantio direto e preparo mínimo do solo. Resultados similares foram obtidos na avaliação da resistência do solo à penetração, independente da época de avaliação e da umidade do solo. Os sistemas de preparo mínimo e preparo convencional do solo proporcionam condições físicas menos restritivas ao crescimento das plantas, quando comparado com o plantio direto. Avaliações de médio-longo prazo são necessárias face às mudanças dinâmicas na qualidade física do solo, impostas pelos sistemas de preparo e manejo do solo e da cultura.
\end{abstract}

Palavras-chave: propriedades físicas do solo, estrutura, plantio direto, escarificação, compactação

\section{SOIL BULK DENSITY, POROSITY AND RESISTANCE TO ROOT PENETRATION IN AN OXISOL MANAGED BY DIFFERENT SOIL TILLAGE SYSTEMS}

\begin{abstract}
Soil tillage and management affect soil physical conditions for plant growth and crop productivity. The objective of this research was to evaluate some soil physical properties of an Typic Haplorthox located in the Northwest region of the State of Paraná, Brazil, after two years of different tillage systems, with same crop. The tillage systems used were: No-tillage (cultivated only along the crop row - NT), Minimum Tillage (subsoiling to the $0.30 \mathrm{~m}$ depth, followed by leveling - MT) and Conventional Tillage (plowing with moldboard plow to the $0.25 \mathrm{~m}$ depth, followed by leveling - CT). The following soil physical properties were evaluated at the depths of $0-0.10 \mathrm{~m}$ and $0.10-0.20 \mathrm{~m}$ : soil bulk density $(\mathrm{Bd})$, soil porosity, and the soil resistance to root penetration (SRP), which was evaluated at each $0.05 \mathrm{~m}$ up to $0.4 \mathrm{~m}$, during three different periods. The results suggest showed higher values of $\mathrm{Bd}$ and smaller values of macroporosity in the 0-0,10 m layer for NT and MT. Similar results were obtained for SRP, independently of the time of evaluation and soil water content. MT and CT may provide favorable soil physical conditions to the growth of the crop when compared to NT. Medium to long-term evaluations are necessary due to the dynamic changes in soil physical quality, result of the tillage system, soil and crop management.

Key words: soil physical properties, soil structure, no-tillage, subsoiling, compaction
\end{abstract}

\section{INTRODUÇÃO}

Os solos agrícolas funcionam como um sistema complexo que retém e transmite água, ar, nutrientes e calor às sementes e plantas, de maneira que é fundamental um ambiente físico favorável ao crescimento radicular, para maximizar a produção das culturas (Letey, 1985; Hamblin, 1985). Neste contexto, os sistemas de preparo do solo devem oferecer condições favoráveis ao crescimento e desenvolvimento das culturas. No entanto, dependendo do solo, do clima, da cultura e de seu manejo, eles podem promover a degradação da qualidade física do solo, com restrições ao crescimento radicular (Klute, 1982).

Nas regiões tropicais, sistemas de preparo com mínima perturbação do solo e que propiciem a manutenção de resíduos na superfície são necessários para o controle da erosão, redução da degradação do 
solo e do meio ambiente (Lal, 2000). O controle da erosão é fundamental para o reduzir o processo de degradação do solo e práticas eficientes exigem a manutenção da cobertura do solo.

A mandioca (Manihot esculenta, Crantz) é freqüentemente cultivada em solos arenosos, geralmente com baixos teores de nutrientes e de matéria orgânica. No Estado do Paraná, esta cultura ocupa atualmente uma área de 160.000 ha, proporcionando uma produção anual de aproximadamente 3,5 milhões de toneladas de raízes tuberosas, com uma produtividade média de 21,875 t ha-1 (Groxko, 1999). Entre os fatores relacionados ao solo, o sistema de manejo e, especificamente, o preparo do solo, interfere diretamente nos níveis de produtividade da cultura da mandioca (Souza, 1986).

Na região Noroeste do Paraná, principal pólo produtor do estado, a mandioca é cultivada utilizando diferentes sistemas de preparo. O sistema de preparo convencional (aração + gradagem) é mais comumente utilizado, por questões tecnológicas. No entanto, devido à suscetibilidade dos solos de textura arenosa à erosão, bem como às elevadas perdas de matéria orgânica, tem sido estimulado a adoção de preparos conservacionistas para manter a cobertura e reduzir a erosão. Assim, o preparo mínimo com escarificação do solo e o plantio direto com revolvimento na linha de plantio, têm se apresentado como alternativas tecnicamente viáveis (Oliveira et al., 2001).

Os efeitos de sistemas de preparo de solo sobre as culturas de raízes tuberosas não são muito estudados, mas tem-se procurado definir o melhor sistema de preparo para um solo em particular (Howeler et al., 1993). Ohiri \& Ezumah (1990) compararam sistemas de preparo baseados principalmente em parâmetros da cultura (produção de raízes, da parte aérea e da produtividade). Outros autores fizeram suas avaliações em função da fertilidade do solo (Cadavid et al., 1998) ou efeitos conjuntos sobre a fertilidade e propriedades físicas do solo (Oliveira, 1998). Os resultados de pesquisa regional mostram que a cultura responde negativamente à redução no revolvimento do solo (Oliveira, 1998; Oliveira et al., 2001), com menor crescimento da parte aérea e da produção de raízes tuberosas. Estudos conduzidos em outros países produtores de mandioca, principalmente na África, confirmam o menor desempenho da cultura com a redução do preparo do solo (Kayombo \& Lal, 1994). A resposta negativa das culturas aos sistemas de plantio com reduzida mobilização do solo tem sido atribuída às condições físicas do solo menos favoráveis ao crescimento das raízes e da parte aérea das plantas.

Os sistemas de preparo do solo promovem modificações nas propriedades físicas como a agregação do solo (Castro Filho et al., 1998), a densidade e a porosidade do solo (De Maria et al., 1999). A macroporosidade é drasticamente reduzida com a compactação do solo (Dias Jr. \& Pierce, 1996). De forma geral estas propriedades funcionam como indicadores de possíveis restrições ao crescimento radicular das culturas.

A resistência do solo à penetração é uma das propriedades físicas do solo diretamente relacionados com o crescimento das plantas (Letey, 1985) e modificada pelos sistemas de preparo do solo. O crescimento das raízes pode causar a deformação do solo numa zona próxima à ponta das raízes e a pressão exercida contra as partículas e/ou agregados deve ser suficiente para propiciar a penetração e 0 alongamento das raízes (Bennie, 1996). Valores excessivos de resistência do solo à penetração podem influenciar 0 crescimento das raízes em comprimento e diâmetro (Merotto \& Mundstock, 1999) e na direção preferencial do crescimento radicular (lijima \& Kono, 1991). Além disso, estudos recentes indicam que a resistência do solo à penetração das raízes tem efeitos diretos no crescimento da parte aérea das plantas (Masle \& Passioura, 1987) e na partição de carboidratos entre a raiz e parte aérea (Masle \& Farquhar, 1988). Valores críticos de resistência à penetração dependem da espécie (Bengough \& Mullins, 1990). Desta forma, a resistência do solo à penetração é fundamental para a avaliação dos efeitos dos sistemas de preparo no ambiente físico do solo para o crescimento das plantas.

A avaliação das propriedades físicas sob diferentes sistemas de preparo do solo é importante para caracterizar o ambiente físico para o crescimento radicular. O objetivo deste estudo é o de avaliar algumas propriedades físicas de um Latossolo Vermelho distrófico cultivado com mandioca, após dois anos de implantação de diferentes sistemas de preparo do solo.

\section{MATERIAL E MÉTODOS}

O experimento foi instalado em Araruna ( $23^{\circ} 54^{\prime}$ $00 \mathrm{~S}, 52^{\circ} 30^{\prime} 00 \mathrm{~W}$ ) , Noroeste do Estado do Paraná, durante o ano agrícola 1999/2000, num Latossolo Vermelho distrófico (Embrapa, 1999). A caracterização granulométrica e química do solo realizada antes da implantação dos sistemas de preparo encontra-se na Tabela 1. O clima da região é mesotérmico sem estação seca, com verões quentes, com temperatura anual média de $21,5^{\circ} \mathrm{C}$, precipitação média anual de $1.617 \mathrm{~mm} \mathrm{e}$ umidade média anual de 62\% (Godoy et al., 1976).

Os tratamentos utilizados constituíram-se de três sistemas de preparo de solo: Plantio Direto - PD; Preparo Mínimo (escarificação, seguido de gradagem niveladora e posterior plantio) - PM; e Preparo Convencional (aração, com arado de aiveca, a uma profundidade entre 0,25-0,30 m, seguido de gradagem niveladora) - PC. Cada parcela experimental tem área total $147 \mathrm{~m}^{2}(7,0 \mathrm{~m}$ x 21,0 m) e área útil de $30 \mathrm{~m}^{2}(2,0 \mathrm{~m} \times 15,0 \mathrm{~m})$. O delineamento experimental utilizado foi o de blocos completos casualizados, com oito repetições, perfazendo 
um total de 24 unidades experimentais. Tanto as parcelas quanto os blocos foram espaçados entre si por distâncias de $1,0 \mathrm{~m}$.

A implantação do experimento iniciou-se com o plantio de aveia preta (Avena strigosa, Schreb), em abril de 1999, utilizando semeadura direta. Quando da floração plena da aveia, a mesma passou por um processo de rolagem por meio de um "rolo-faca". Este procedimento foi realizado também antes da introdução do segundo cultivo de mandioca na safra 2000/2001.

Para a determinação das propriedades físicas do solo, foram retiradas amostras com estrutura indeformada, utilizando amostrador e anéis com volume de $100 \mathrm{~cm}^{3}$, no centro das camadas de 0-0,10 m e 0,10-0,20 m. Após a coleta, as amostras foram acondicionadas em sacos plásticos e mantidas sob temperatura de $\pm 5^{\circ} \mathrm{C}$ até serem processadas.

A densidade do solo foi determinada de acordo com Blake \& Hartge (1986). Para a determinação da porosidade do solo, as amostras indeformadas foram saturadas por 48 horas em bandeja com água até dois terços da altura do anel. Após o período de saturação, as amostras foram drenadas no potencial equivalente a $-0,006 \mathrm{MPa}$ utilizando uma mesa de tensão adaptada de Kiehl (1979). A partir dos valores de umidade com saturação da amostra e dos valores de retenção de água, calculou-se os valores de macro, micro e porosidade total do solo.

A resistência do solo à penetração foi realizada conforme Tormena \& Roloff (1996), obtendo-se dados de resistência à penetração a cada $0,05 \mathrm{~m}$ até $0,40 \mathrm{~m}$ de profundidade, utilizando um penetrômetro modelo Solotest. As amostragens de resistência à penetração foram feitas em diferentes épocas para avaliar o nível de resistência do solo imposto pelos sistemas de preparo sob diferentes condições de umidade do solo e avaliar os impedimentos impostos pelos sistemas de preparo à cultura. Em cada parcela experimental obteve-se três medidas de resistência e um valor médio de cada profundidade foi utilizado para representar a parcela individualmente.

Para as variáveis densidade e porosidade do solo, a análise dos dados foi feita conforme Hatcher \& Stepanski (1997). Constatando-se efeito significativo da interação entre tratamento e camada amostrada $(P<$
0,05), procederam-se os desdobramentos necessários e aplicou-se 0 teste de Tukey $(P<0,05)$, para a comparação das médias das variáveis analisadas.

Os dados de resistência à penetração foram avaliados e analisados utilizando um valor médio em cada profundidade. $O$ erro padrão da média foi utilizado para proceder à avaliação dos tratamentos em cada profundidade amostrada. Adotou-se o critério de Gravetter \& Wallnau (1995) para diferenciar estatisticamente os tratamentos, o que ocorre quando não há sobreposição dos limites superior e inferior dos erros padrão na comparação das médias dos tratamentos.

As análises estatísticas foram conduzidas utilizando o programa estatístico SAS (SAS Institute, 1999).

\section{RESULTADOS E DISCUSSÃO}

\section{Densidade do solo}

O correu interação $(P<0,05)$ entre os tratamentos e as camadas avaliadas. Os valores médios da densidade do solo nas camadas de 0-0,10 m e 0,100,20 m estão mostrados na Figura 1. Na camada de 0,10-0,20 m não foi constatado efeito dos sistemas de preparo $(P>0,05)$.

$\mathrm{Na}$ camada de 0-0,10 m constataram-se diferenças entre os tratamentos. A exemplo da camada de $0,10-0,20 \mathrm{~m}$, o maior valor de densidade foi obtido no PD, o que se deve ao efeito cumulativo do tráfego de máquinas, da ausência de mobilização mecânica do solo e, conforme Voorhees (1983), da ineficiência das forças naturais (ciclos de secagem e umedecimento) em reduzir a densidade do solo. No PM os valores de densidade do solo são intermediários entre PC e PD, e diferentes de ambos, sendo associados ao revolvimento parcial do solo pelo equipamento de escarificação. Resultados similares foram obtidos por Onwualu \& Anazodo (1989) num Oxissolo na Nigéria. Os menores valores da densidade do solo podem ser atribuídos ao intenso revolvimento do solo e à incorporação dos resíduos culturais (Hill, 1990). O maior valor de densidade do solo no PD, comparado com outros sistemas de preparo tem sido constatado por outros autores em estudos conduzidos no Brasil (Centurion \& Demattê, 1985; Klepker \& Anghinoni,

Tabela 1 - Características químicas e granulometria do Latossolo Vermelho distrófico nas camadas.

\begin{tabular}{|c|c|c|c|c|c|c|c|c|c|c|c|}
\hline Camada & $\mathrm{pH} \mathrm{CaCl}{ }_{2}$ & $\mathrm{Al}^{+3}$ & $\mathrm{H}^{+}+\mathrm{Al}^{+3}$ & $\mathrm{Ca}^{+2}+\mathrm{Mg}^{+2}$ & $\mathrm{Ca}^{+2}$ & $\mathrm{~K}^{+}$ & $\mathrm{P}$ & $\mathrm{C}$ & areia & silte & argila \\
\hline --- m --- & & -..- & - & $\mathrm{mmol}_{\mathrm{c}} \mathrm{dm}^{-3}$ & - & - & $g d m$ & $\mathrm{~g} \mathrm{dm}^{-3}$ & - & $\mathrm{kg}^{-1}-$ & $\ldots$ \\
\hline $0,0-0,1$ & 5,4 & 0 & 33,1 & 37,4 & 22,8 & 6,0 & 9,0 & 10,8 & 680 & 10 & 310 \\
\hline $0,1-0,2$ & 5,0 & 2,7 & 37,0 & 35,0 & 23,2 & 4,9 & 4,7 & 11,4 & 690 & 10 & 300 \\
\hline $0,2-0,4$ & 4,5 & 6,0 & 43,4 & 22,5 & 14,8 & 1,7 & 1,0 & 5,8 & 650 & 20 & 330 \\
\hline $0,4-0,6$ & 4,5 & 7,7 & 48,1 & 18,7 & 13,7 & 0,7 & 1,0 & 4,8 & 650 & 20 & 330 \\
\hline $0,6-0,8$ & 4,2 & 12,7 & 57,7 & 13,9 & 9,8 & 0,5 & 1,0 & 6,1 & 630 & 10 & 360 \\
\hline $0,8-1,0$ & 4,2 & 12,7 & 59,0 & 14,5 & 10,6 & 0,4 & 0,7 & 6,0 & 630 & 30 & 340 \\
\hline
\end{tabular}


1995; Corsini \& Ferraudo, 1999) e em outros países (Onwualu \& Anazodo, 1989; Voorhees, 1983). Sob condições de maior densidade do solo na superfície, há possibilidade de reduções na produtividade das culturas quando da ocorrência de déficit hídrico nos estágios fenológicos críticos (Domzal \& Slowinska-Jurkiewicz, 1987), o que foi constatado por Onwualu \& Anazodo (1989). A longo prazo, é possível que o acúmulo de matéria orgânica e a redução no tráfego contribuam para reduzir a densidade do solo no sistema de plantio direto.

As diferenças de densidade do solo entre as duas camadas avaliadas revelam os efeitos dos implementos no fracionamento dos agregados com conseqüente criação de poros nos sistemas PC e PM. A estrutura do solo após a aplicação dos implementos de preparo é fortemente dependente do tipo de implemento utilizado, das condições climáticas e da umidade do solo (Domzal \& Slowinska-Jurkiewicz, 1987).

\section{Porosidade do solo}

Houve interação $(P<0,05)$ entre tratamento e camada para as variáveis macroporosidade e porosidade total e efeitos de camada e tratamento para a variável microporosidade $(P<0,05)$. Em concordância com as indicações de Dias Jr. \& Pierce (1996), a macroporosidade foi a variável mais afetada pelos tratamentos, o que foi constatado pelos maiores valores de F obtidos na ANOVA.

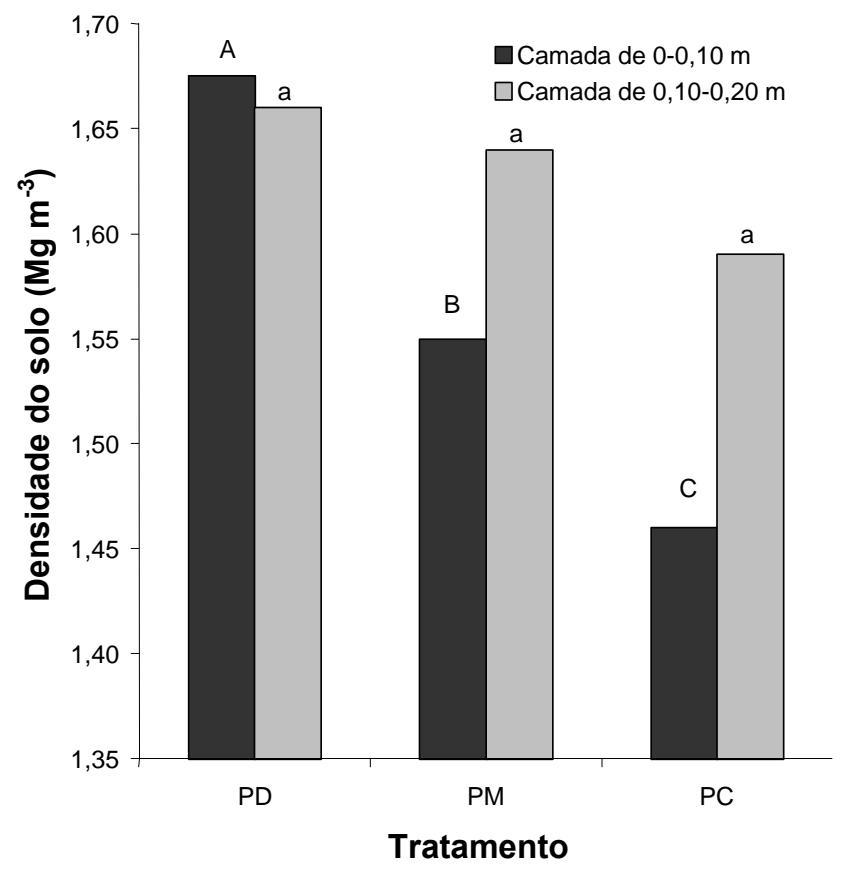

Figura 1 - Valores médios de densidade do solo nos sistemas de preparo e camadas amostradas. As letras maiúsculas $(0-0,10 \mathrm{~m})$ e minúsculas $(0,10-0,20 \mathrm{~m})$ diferentes indicam que as médias dos tratamentos são diferentes (Tukey, $P$ $<0,05)$. $P D=$ plantio direto; $P M=$ preparo mínimo; $P C=$ preparo convencional.
Os sistemas de preparo PM e PC promoveram aumentos na porosidade do solo, quando comparados ao sistema PD (Tabela 2). A utilização do arado e de escarificadores, nos tratamentos PC e PM, proporcionaram os maiores valores $(P<0,001)$ de macroporosidade e porosidade total comparado com 0 PD. Em relação à microporosidade, houve aumento no PD comparado ao PC, o que se deve ao aumento da densidade do solo no PD. Os maiores valores de macroporosidade nos sistemas com revolvimento, até o período da avaliação deveram-se à persistência dos efeitos da mobilização do solo que resultaram em fraturamento dos agregados e o desenvolvimento de poros, notadamente macroporos. Os efeitos do revolvimento foram mais importantes na superfície do solo, visto o decréscimo no volume de macroporos, em profundidade, no PM e PC, aproximando aos valores do PD. No PC e PM a redução do volume de macroporos em profundidade foi de $64,6 \%$ e $59,5 \%$, respectivamente. No PD os valores de macroporosidade não variaram em profundidade (Tabela 2). O aumento da densidade do solo no PD (Figura 1) ocorreu às expensas dos poros de maior diâmetro.

Os resultados obtidos estão em conformidade com aqueles de Hill \& Cruse (1985), os quais constataram um aumento na macroporosidade com o revolvimento do solo. O plantio direto, além de reduzir a porosidade total, muda drasticamente a distribuição do tamanho dos poros, com redução dos poros de maior tamanho (Van Ouwerkek \& Boone, 1970). Desta forma, a hipótese de que o revolvimento do solo aumenta o número de poros drenáveis (Tollner et al., 1984) também é confirmada pelo aumento no volume de macroporos no PC e PM. As implicações destes resultados, conforme Hill \& Cruse (1985), são de que o solo sob preparo convencional poderia drenar mais rapidamente do que aquele com menor intensidade de mobilização e influenciar temporalmente a disponibilidade de água às plantas.

Tabela 2 - Valores médios de macroporosidade (Ma), microporosidade e porosidade total $(\mathrm{Pt})$ nos sistemas de preparo e camadas estudadas. As letras diferentes na vertical indicam que as médias dos tratamentos são diferentes (Tukey, $P<0,05)$. PD = plantio direto; $P M=$ preparo mínimo; $\mathrm{PC}=$ preparo convencional.

\begin{tabular}{|c|c|c|c|c|}
\hline \multicolumn{3}{|c|}{ Camada de $0-0,10 \mathrm{~m}$} & \multicolumn{2}{|c|}{ Camada de $0,10-0,20 \mathrm{~m}$} \\
\hline Preparo & $\mathrm{Ma}$ & $\mathrm{Pt}$ & $\mathrm{Ma}$ & $\mathrm{Pt}$ \\
\hline \multicolumn{5}{|c|}{ - } \\
\hline $\mathrm{PC}$ & $0,1698 \mathrm{~A}$ & $0,4500 \mathrm{~A}$ & $0,1097 \mathrm{~A}$ & $0,4025 \mathrm{~A}$ \\
\hline $\mathrm{PM}$ & $0,1436 \mathrm{~A}$ & $0,4263 \mathrm{~A}$ & $0,0855 \mathrm{~A}$ & $0,3875 \mathrm{~A}$ \\
\hline PD & $0,0813 \mathrm{~B}$ & $0,3713 \mathrm{~B}$ & $0,0846 \mathrm{~A}$ & $0,3875 \mathrm{~A}$ \\
\hline Preparo & \multicolumn{4}{|c|}{ Microporosidade $\left(\mathrm{m}^{3} \mathrm{~m}^{-3}\right)$} \\
\hline $\mathrm{PC}$ & $0,285 \mathrm{~A}$ & \multicolumn{2}{|c|}{ Camada (m) } & \\
\hline PM & $0,293 \mathrm{AB}$ & \multicolumn{2}{|c|}{$0-0,10$} & $0,2845 \mathrm{a}$ \\
\hline PD & $0,296 \mathrm{~B}$ & \multicolumn{2}{|c|}{$0,10-0,20$} & $0,2992 b$ \\
\hline
\end{tabular}


A macroporosidade é uma medida diretamente relacionada com a difusão de oxigênio no solo para as raízes (Grable \& Siemer, 1968). A macroporosidade determina a capacidade de aeração do solo (Thomasson, 1978 e Erickson, 1982), e os resultados obtidos sugerem que a capacidade de aeração do solo segue em ordem crescente nos sistemas de preparo $\mathrm{PD}<\mathrm{PM}<\mathrm{PC}$. Valores de porosidade de aeração abaixo de $10-15 \%$ são, geralmente, adotados como restritivos para o crescimento e produtividade da maioria das culturas, apesar da dependência da espécie de planta e da atividade biológica do solo. Valores adequados de capacidade de aeração são dependentes das condições climáticas e os valores críticos de porosidade de aeração devem ser ampliados sob condições mais úmidas (Thomasson, 1978).

\section{Resistência do solo à penetração}

A resistência do solo à penetração (RP) integra os efeitos da densidade e da umidade nas condições físicas do solo necessárias para o crescimento das raízes. Os resultados da avaliação da RP em 12/2000 são apresentados na Figura 2 e as respectivas umidades a base de massa, são mostradas na Figura 3. Há tendência de redução da resistência à penetração em profundidade, o que se deve ao efeito do secamento do solo em superfície (Figura 3). Constataram-se maiores valores de resistência do solo à penetração no PD comparado ao PC e PM, até a profundidade de $0,25 \mathrm{~m}$. Os menores valores de RP no PC comparados ao PM devem-se ao revolvimento parcial do solo pelo escarificador, visto que a umidade do solo é maior no PM do que no PC. Estes resultados concordam com os obtidos por Wutke et al. (2000), que indicam o efeito da escarificação na redução da densidade do solo.

De acordo com Watanabe (2001) a umidade deste solo na capacidade de campo (CC) é de $0,165 \mathrm{~kg}$ $\mathrm{kg}^{-1}$. A avaliação conduzida em 12/2000 foi realizada com umidade do solo abaixo da CC, e em processo de secamento, o que justifica os elevados valores de RP, acima do valor crítico de 2,0 MPa (Bengough \& Mullins, 1990) até $0,10 \mathrm{~m}$ de profundidade.

Com o aumento da umidade em profundidade ocorre, simultaneamente, a redução da RP. Apesar da semelhança dos valores de umidade entre PC e PD, a maior resistência em $P D$ é resultado da maior compactação do solo, com maior densidade e menor volume de macroporos. Nas camadas superficiais, sob PD, a RP atinge níveis altamente impeditivos ao crescimento das plantas. Por exemplo, os resultados obtidos por Merotto \& Mundstock (1999) indicam que RP maior que 3,5 MPa causa severa limitação ao crescimento de plantas de trigo. Os maiores valores de RP no PD podem influenciar a produtividade de raízes, uma vez que afetam diretamente o crescimento das raízes e parte aérea (Masle \& Passioura, 1987) e indiretamente devido a um deficiente fornecimento de água e nutrientes (Atwel, 1990).
Os resultados da avaliação executada em 02/ 2001 são mostrados na Figura 4. Em comparação com os resultados obtidos em 12/2000, esta avaliação foi realizada com solo sob umidade próxima da CC (umidade média igual a $0,164 \mathrm{~kg} \mathrm{~kg}^{-1}$ até $0,20 \mathrm{~m}$ e 0,158 $\mathrm{kg} \mathrm{kg}^{-1}$ de $0,20-0,40 \mathrm{~m}$ ) em todos os tratamentos. Em comparação com a amostragem feita em 12/200 os

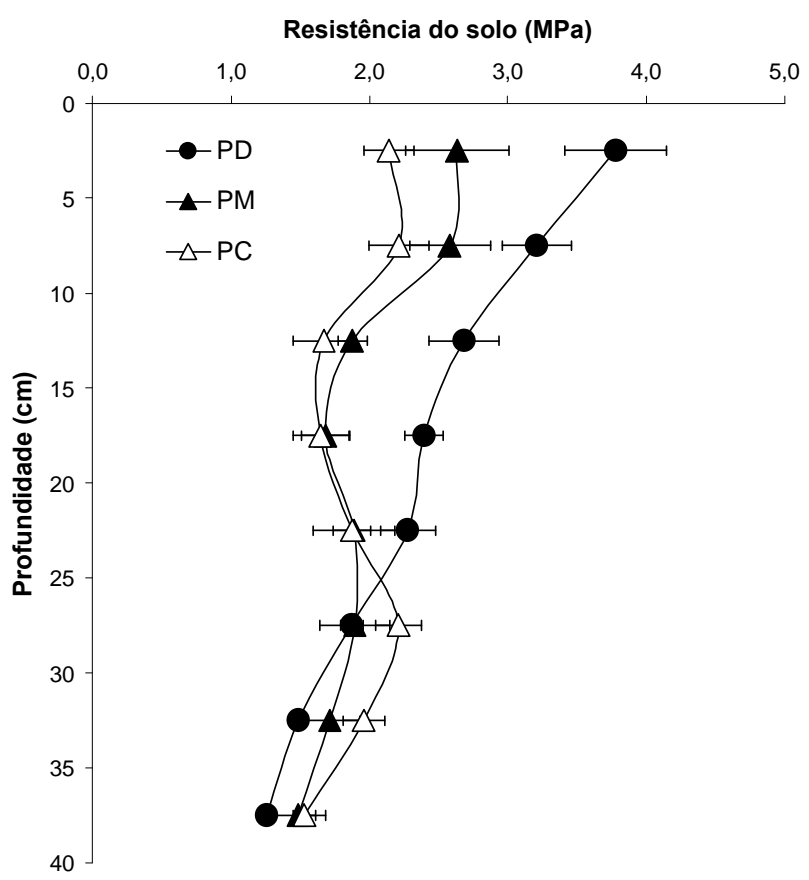

Figura 2 - Resistência do solo à penetração na amostragem realizada em dezembro de 2000. As barras indicam os valores de erro padrão da média e a sobreposição destas denota a ausência de diferenças entre as médias dos tratamentos. $\mathrm{PD}=$ plantio direto; $\mathrm{PM}=$ preparo mínimo; $\mathrm{PC}=$ preparo convencional.

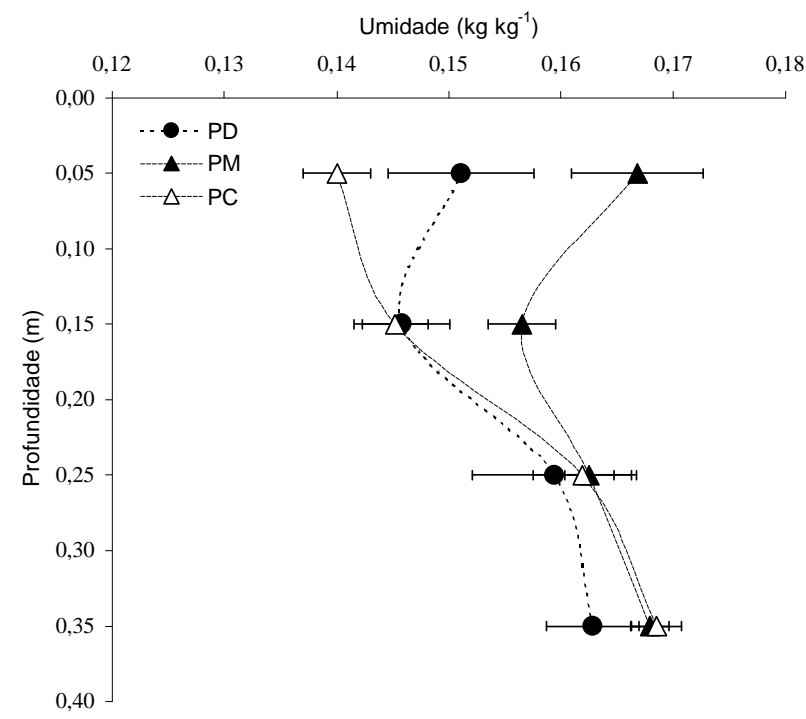

Figura 3 - Umidade do solo na amostragem realizada em dezembro de 2000. As barras indicam os valores de erro padrão da média e a sobreposição destas denota a ausência de diferenças entre as médias dos tratamentos. $P D=$ plantio direto; $\mathrm{PM}=$ preparo mínimo; $\mathrm{PC}=$ preparo convencional. 
valores de RP foram substancialmente reduzidos, indicando o efeito da umidade do solo em reduzir a RP (Tormena \& Roloff, 1996).

No solo sob condições mais úmidas, os valores de resistência à penetração podem ser considerados não impeditivos para os sistemas PC e PM e pouco impeditivos para o sistema PD. No entanto, com 0 secamento do solo os valores de resistência podem atingir níveis altamente impeditivos, especialmente sob PD.

Os resultados da avaliação da RP realizada em 06/2001 são apresentados na Figura 5. Esta avaliação também foi executada com solo próximo à $\mathrm{CC}$, condição de umidade similar àquela realizada em 02/2001. $O$ sistema de PD apresentou valores de RP maiores que o PC e PM, sendo o PC menor do que o PM. Sob condições de elevada umidade do solo, a resistência é drasticamente reduzida e, como constatado na avaliação anterior, valores maiores que 2,0 MPa apenas ocorreram no PD até $0,25 \mathrm{~m}$ de profundidade. Estes resultados estão de acordo com os obtidos por Tormena \& Roloff (1996).

Os resultados obtidos neste estudo estão de acordo com aqueles apresentados por Watanabe (2001) que, em condições semelhantes, constatou maiores valores de resistência do solo à penetração sob PD. De Maria et al. (1999) também constataram maiores valores de RP sob plantio direto, em comparação com preparo do solo com escarificação até $0,30 \mathrm{~m}$ de profundidade. A mobilização do solo com arado de aivecas e escarificador reduziu substancialmente a resistência do solo à penetração em comparação com o PD, independente da umidade do solo por ocasião da amostragem. Isto indica que a redução da densidade do solo em PM e PC, constatada na Figura 1, está determinando tanto os níveis de resistência como a sensibilidade do solo em aumentar a resistência com a perda de água. Estes resultados sugerem a necessidade de realizar pesquisas com maior tempo de implantação dos sistemas de preparo.

Em termos de redução da densidade do solo e da resistência do solo à penetração, o PC mostrou-se mais eficiente que o PM em relação ao PD. Considerando a necessidade de cobertura do solo para controle da erosão e das perdas de água, os benefícios físicos do PC podem ser anulados pela rápida perda de água do solo, em face de maior volume de poros drenáveis no PC. Isto torna as culturas mais vulneráveis às condições climáticas neste sistema de preparo. Os resultados indicam que na camada de $0-0,10 \mathrm{~m}$ a densidade do solo foi reduzida com a adoção de sistemas de preparo com revolvimento do solo. No PD foram observados os maiores valores de densidade do solo e os menores valores de macroporosidade. $\mathrm{Na}$ camada de 0,10-0,20 m não foi constatado efeito significativo dos sistemas de preparo na densidade e porosidade do solo. Sob PD, os valores de resistência à penetração são maiores que PC e PM, independentemente da umidade do solo. Os resultados de resistência à penetração sugerem que, no processo de secamento do solo sob PD, ocorre um aumento expressivo da resistência à penetração das raízes no mesmo.

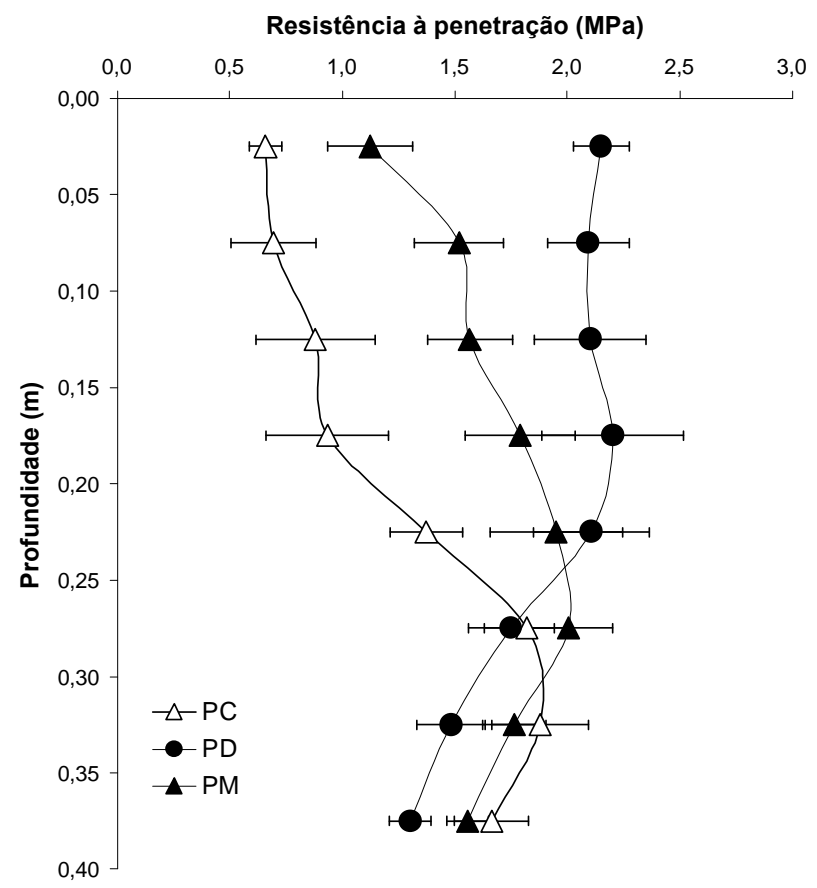

Figura 4 - Resistência do solo à penetração na amostragem realizada em fevereiro de 2001. As barras indicam os valores do erro padrão da média e a sobreposição destas denota a ausência de diferenças entre as médias dos tratamentos. $\mathrm{PD}=$ plantio direto; $\mathrm{PM}=$ preparo mínimo; $\mathrm{PC}=$ preparo convencional.

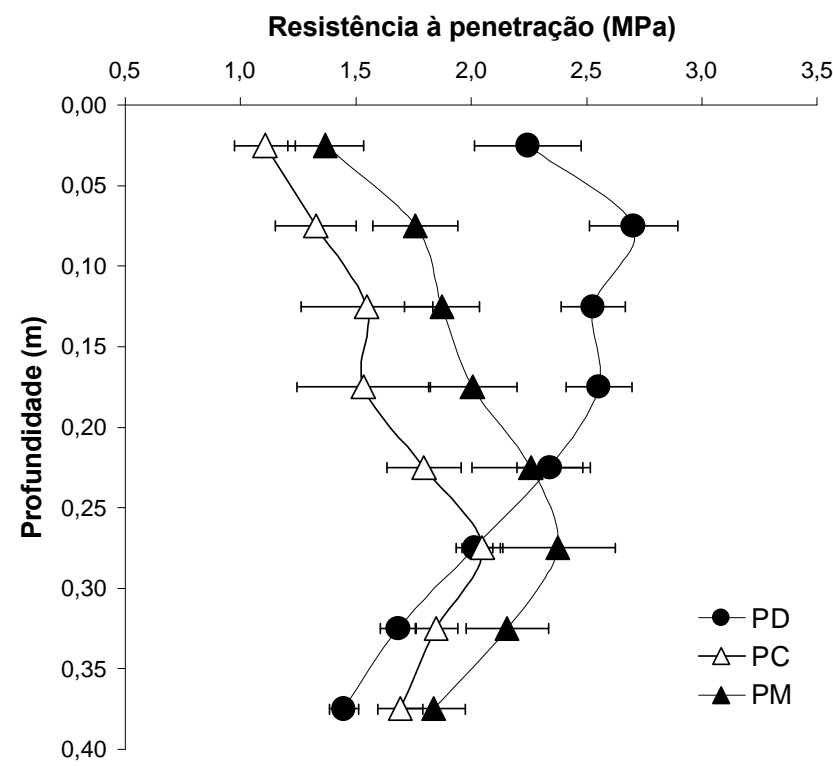

Figura 5 - Resistência do solo à penetração nos três diferentes sistemas de preparo adotados, na amostragem realizada em 06/2001. As barras indicam os valores do erro padrão da média e a sobreposição destas denota a ausência de diferenças entre as médias dos tratamentos. $P D=$ plantio direto; $\mathrm{PM}=$ preparo mínimo; $\mathrm{PC}=$ preparo convencional. 


\section{REFERÊNCIAS BIBLIOGRÁFICAS}

ATWELL, B.J. The effect of soil compaction on wheat during early tillering. I. Growth, development and root structure. New Phytologist, v.115, p.2935, 1990.

BENGHOUGH, A.G.; MULLINS, C.E. Mechanical impedance to root growth: a review of experimental techniques and root growth responses. Journal of Soil Science, v.41, p.341-358, 1990

BENNIE, A.T.P. Growth and mechanical impedance. In: WAISEL, Y.; ESHEL, A.; KAFKAFI, U. (Ed.) Plant roots: the hidden half. 2.ed. New York: Marcel Dekker, 1996. p.453-470.

BLAKE, G.R.; HARTGE, K.H. Bulk density. In: KLUTE, A. (Ed.) Methods of soil analysis: physical and mineralogical methods. Madison: ASA, 1986. cap.13, p.363-375

CADAVID, L.F.; EL-SHARKAWY, M.A.; ACOSTA, A.; SÁNCHEZ, T. Long-term effects of mulch, fertilization and tillage on cassava grown in sandy soils in northern Colombia. Field Crops Research, v.57, p.45-56, 1998.

CASTRO FILHO, C.; MUZILLI, O.; PODANOSCHI, A.L. Estabilidade de agregados e sua relação com o teor de carbono orgânico num latossolo roxo distrófico, em função de sistemas de plantio, rotação de culturas e métodos de preparo das amostras. Revista Brasileira de Ciência do Solo, v.22, p.527-538, 1998.

CENTURION, J.F.; DEMATTÊ, J.L.I. Efeito de sistemas de preparo nas propriedades físicas de um solo sob cerrado cultivado com soja. Revista Brasileira de Ciência do Solo, v.9, p.263-266, 1985.

CORSINI, P.C.; FERRAUDO, A.S. Efeitos de sistemas de cultivo na densidade e macroporosidade do solo e no desenvolvimento radicular do milho em Latossolo Roxo. Pesquisa Agropecuária Brasileira, v.34, p.289-298, 1999.

De MARIA, I.C.; CASTRO, O.M.; DIAS, H.S. Atributos físicos do solo e crescimento radicular de soja em latossolo roxo sob diferentes métodos de preparo do solo. Revista Brasileira de Ciência do Solo, v.23, p.703709, 1999

DIAS JR., M.S.; PIERCE, F.J. O processo de compactação do solo e sua modelagem. Revista Brasileira de Ciência do Solo, v.20, p.175-182, 1996.

DOMZAL, A ; SLOWINSKA-JURKIEWICZ, A. Effect of tillage and wheather condition on the structure and physical properties of soil and yield of winter wheat. Soil \& Tillage Research, v.10, p.225-241, 1987.

EMPRESA BRASILEIRA DE PESQUISA AGROPECUÁRIA. Serviço Nacional de Levantamento e Conservação dos Solos. Sistema brasileiro de classificação de solos. Brasília: Embrapa Produção de Informação, 1999. $412 p$.

ERICKSON, A.E. Tillage effects on soil aeration. In: VAN DOREN, D.M.; ALLMARAS, R.R.; LINDEN, D.R.; WHISLER, F.D. (Ed.) Predicting tillage effects on soil physical properties and processes. Madison: ASA, 1982. cap.6, p.91-104.

GODOY, H.; CORREA, A.R.; SANTOS, D. Clima do Paraná. In: FUNDAÇÃO INSTITUTO AGRONOMICO DO PARANÁ. Manual agropecuário para o Paraná. Londrina, 1976. 178p.

GRABLE, A.R.; SIEMER, E.G. Effects of bulk density, aggregate size, and soil water suction on oxygen diffusion, redox potential and elongation of corn roots. Soil Science Society of America Journal, v.32, p.180-186, 1968.

GRAVETTER, F.J.; WALLNAU, L.B. Statistics for the behavioral sciences. 2.ed. St. Paul: West Publishing, 1995. 429p.

GROXKO, M. Mandioca. Acompanhamento da Situação Agropecuária no Paraná. Curitiba, v.25, p.39-45, 1999.

HAMBLIN, A.P. The influence of soil structure on water movement, crop root growth and water uptake. Advances in Agronomy, v.38, p.95-158, 1985.

HATCHER, L.; STEPANSKI, E.J. A step-by-step approach to using the SAS System for Univariate and Multivariate Statistics. Cary: SAS Institute Inc., 1997. 552p.

HILL, R.L. Long-term conventional and no-tillage effects on selected soil physical properties. Soil Science Society of America Journal, v.54, p.161-166, 1990

HILL, R.L.; CRUSE, R.M. Tillage effects on bulk density and soil strength of two Mollisols. Soil Science Society of America Journal, v.49, p.12701273, 1985.

HOWELER, R.H.; EZUMAH, H.C.; MIDMORE, D.J. Tillage systems for root and tuber crops in the tropics. Soil \& Tillage Research, v.27, p.211-240, 1993.
IIJIMA, M.; KNO, Y. Interspecific differences of the root system structures of four cereal species as affected by soil compaction. Japanese Journal of Crop Science, v.60, p.130-138, 1991

KAYOMBO, B.; LAL, R. Responses of tropical crops to soil compaction. In: SOANE, B.D.; VAN OUWERKERK, C. (Ed.) Soil compaction in crop production. Amsterdan: Elsevier Science, 1994. cap.13, p.287-316.

KIEHL, E.J. Manual de edafologia - relações solo-planta. São Paulo: Ed. Agronômica Ceres, 1979. 262p.

KLEPKER, D.; ANGHINONI, I. Características físicas e químicas do solo afetadas por métodos de preparo e modos de adubação. Revista Brasileira de Ciência do Solo, v.19, p.395-401, 1995.

KLUTE, A. Tillage effects on the hydraulic properties of soil: a review. In: VAN DOREN, D.M.; ALLMARAS, R.R.; LINDEN, D.R.; WHISLER, F.D. (Ed.) Predicting tillage effects on soil physical properties and processes. Madison: ASA, 1982. cap.3, p.29-43.

LAL, R. Soil management in the developing countries. Soil Science, v.165, p.57-72, 2000

LETEY, J. Relationship between soil physical properties and crop production Advances in Soil Science, v.1, p.277-294. 1985.

MASLE, J.; FARQUHAR, G.D. Effects of soil strength on the relation of wateruse-efficiency and growth to carbon isotope discrimination in wheat seedlings. Plant Physiology, v.86, p.32-38, 1988

MASLE, J.; PASSIOURA, J.B. The effect of soil strength on the growth of young wheat plants. Australian Journal of Plant Physiology, v.14, p.643 656, 1987

MEROTTO, A.; MUNDSTOCK, C.M. Wheat root growth as affected by soil strength. Revista Brasileira de Ciência do Solo, v.23, p.197-202, 1999

OHIRI, A.C. EZUMAH, H.C. Tillage effects on cassava (Manihot esculenta) production and some soil properties. Soil \& Tillage Research, v.17, p.221229, 1990

OLIVEIRA, J.O.A.P. Efeito de sistemas de preparo em algumas propriedades do solo e na cultura da mandioca (Manihot esculenta, Crantz). Maringá, 1998. 112p. Dissertação (Mestrado) - Universidade Estadual de Maringá

OLIVEIRA, JOA.P. VIDIGAL FILHO, P S ; TORMENA, C A ; PEQUENO, M.G.; SCAPIM, C.A.; MUNIZ, A.S.; SAGRILO, E. Influência de sistemas de preparo do solo na produtividade da mandioca. Revista Brasileira de Ciência do Solo, v.25, p.443-450, 2001

ONWUALU, A.P.; ANAZODO, U.G.N. Soil compaction effects on maize production under various tillage methods in a derived savannah zone of Nigeria. Soil \& Tillage Research, v.14, p.99-114, 1989

SAS. Statistical Analysis System Institute. SAS/STAT Procedure guide for personal computers. Version 5. Cary: SAS Institute, 1999.

SOUZA, L.S. Manejo e conservação do solo para a cultura da mandioca. In: CURSO INTENSIVO NACIONAL DE MANDIOCA, Cruz das Almas, 1986 Cruz das Almas: EMBRAPA, CNPMF, 1986. 40p.

THOMASSON, A.J. Towards an objective classification of soil structure. Journal of Soil Science, v.29, p.38-46, 1978.

TOLLNER, E.W.; HARGROVE, W.L.; LANGDALE, G.W. Influence of conventional and no-tillage practices on soil physical properties in the southern Piedmont. Journal of Soil Water Conservation, v.39, p.73-76 1984.

TORMENA, C.A.; ROLOFF, G. Dinâmica da resistência à penetração de um solo sob plantio direto. Revista Brasileira de Ciência do Solo, v.20, p.333-339, 1996.

VAN OUWERKERK, C.; BOONE, F.R. Soil-physical aspects of zero-tillage experiments. Netherlands Journal of Agricultural Science, v.18, p.247261, 1970.

VOORHEES, W.B. Relative effectiveness of tillage and natural forces in alleviating wheel-induced soil compaction. Soil Science Society of America Journal, v.47, p.129-133, 1983.

WATANABE, S.H. Caracterização da qualidade física de um latossolo vermelho distrófico sob diferentes sistemas de preparo. Maringá, 2001. 69p. Dissertação (Mestrado) - Universidade Estadual de Maringá

WUTKE, E.B.; ARRUDA, F B ; FANCELLI, A.L.; PEREIRA, J.C.V.N.A.; SAKAI, E.; FUJIWARA, M.; AMBROSANO, G.M.B. Propriedades do solo e sistema radicular do feijoeiro irrigado em rotação de culturas. Revista Brasileira de Ciência do Solo, v.24, p.621-633, 2000.

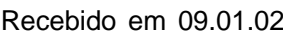

